\title{
Tuberculous Spondylitis, 14 Years' Experience of a Tertiary Care Center in Saudi Arabia
}

\author{
Abdulrahman Balkhoyor', Hind Alhatmi'1, Mohammad Bosaeed1, Abdulrahman Alsaedy1,2, \\ Sami Aleissa ${ }^{2,3}$, Sameera Aljohani' ${ }^{2,4}$, Adel Alothman1,2* \\ ${ }^{1}$ Infectious Disease Division, Department of Medicine, King Abdulaziz Medical City, Riyadh, Saudi Arabia \\ ${ }^{2}$ King Saud bin Abdulaziz University for Health Sciences, Riyadh, Saudi Arabia \\ ${ }^{3}$ Orthopedic Surgery, Department of Surgery, King Abdulaziz Medical City, Riyadh, Saudi Arabia \\ ${ }^{4}$ Microbiology Section, Department of Laboratory Medicine, King Abdulaziz Medical City, Riyadh, Saudi Arabia \\ Email: dr.balkhoyor@gmial.com,dr.hindabdullah@gmail.com, aloufi10@yahoo.com, eissas@ngha.med.sa, \\ johanis@ngha.med.sa, *othmanaf@hotmail.com
}

How to cite this paper: Balkhoyor, A., Alhatmi, H., Bosaeed, M., Alsaedy, A., Aleissa, S., Aljohani, S. and Alothman, A. (2018) Tuberculous Spondylitis, 14 Years' Experience of a Tertiary Care Center in Saudi Arabia. Advances in Infectious Diseases, 8, 229-240.

https://doi.org/10.4236/aid.2018.84019

Received: October 18, 2018

Accepted: November 16, 2018

Published: November 19, 2018

Copyright $(9) 2018$ by authors and Scientific Research Publishing Inc. This work is licensed under the Creative Commons Attribution International License (CC BY 4.0).

http://creativecommons.org/licenses/by/4.0/

\begin{abstract}
Background: Spinal Tuberculosis is the most common and dangerous form of skeletal tuberculosis. It has the potential for serious morbidity, including permanent neurologic deficits and severe deformity. The aim of this study is to review spinal Tuberculosis (TB) cases in our tertiary care center and evaluate it from different aspects, which may provide great support to the clinical decisions of this disease. Methods: The study was a retrospective charts review of all adult spinal TB patients who were evaluated at King Abdulaziz Medical City, Riyadh, from January 2001 to March 2015. The inclusion of cases will need to meet a specific case definition. Patients demography, presenting symptoms and signs, and radiological and microbiological data for all the patients were collected and reviewed. Results: A total of 61 cases screened charts were included according to the case definition. 59\% were female, and the mean age was 51.3 years. The most presenting symptom was back pain (85\%), followed by lower limbs weakness and lower limbs pain. The average duration of symptoms was 170 days. The average hospital stay was 41.4 days. On physical examination, about half of the patients had bilateral lower limbs weakness. Acute phase reactants like C-reactive protein (CRP) and Erythrocyte sedimentation rate (ESR) were elevated, $97 \%$ and $92 \%$ respectively, however leukocytosis was not common. Out of all the patients who had a biopsy done (59 patients), 51 patients (86\%) had positive AFB culture. MTB PCR was done from spine biopsy in 16 patients, and $81 \%$ were positive. Out of 53 tissue specimens, histopathology showed $45 \%$ necrotizing granuloma, $21 \%$ non-necrotizing granuloma, and $34 \%$ non-specific inflammation. Imaging (either CT scan or MRI) was performed on the patients at the time of presentation, and the most common signs seen in both were vertebral bone destruction $(95 \%, 80 \%)$ and perivertebral collections $(70 \%, 84 \%)$. The thoracic
\end{abstract}


and lumbar vertebrae were the most common involved locations. Thirty-one patients had both modalities done (CT and MRI), and spinal compression was seen on MRI in $68 \%$ of the cases compared to $36 \%$ on the CT scan. All the patients received the first line therapy with some individual adjustment and only 39\% (27 patients) underwent surgery. Conclusion: Spinal Tuberculosis is a chronic presentation illness with a non-specific clinical picture. Interventional diagnostics is the best current modality to reach a confirmatory diagnosis. The gold standard for confirming TB diagnosis probably will remain cultures in addition to early utilization of molecular TB diagnostics. Time for diagnosis of Spinal Tuberculosis is delayed despite improvement in diagnostics tests. Medical treatment is the major solution of uncomplicated spinal TB.

\section{Keywords}

Spinal Tuberculosis, Clinical Presentation, Saudi Arabia

\section{Background}

Tuberculosis (TB) is an endemic disease in developing countries. The incidence of spinal TB is not well known, but it is an ancient disease and been identified in Egyptian mummies dating back to $9000 \mathrm{BC}$. TB is found to affect almost all parts of the body [1].

Locally, the estimated TB annual incidence as per latest reports of WHO and Saudi Ministry of health is decreasing compared to previous years. New TB cases in 2016 were 10 per 100,000 population, 6.82 represents the annual incidence rate of Pulmonary TB, and 2.35 is the rate of Non-Pulmonary cases including Skeletal diseases [2] [3]. There are potential underreported cases of presumptive TB when microbiological studies are negative but with compatible clinical and radiological changes and treatment response. Several reviews were done to evaluate this kind of disease in different areas around the globe [4]-[9]. The skeletal TB disease accounts for 10 to 35 percent of all extrapulmonary tuberculosis cases [10] [11] [12] [13] [14]. Among the common presentation of skeletal TB is Pott's disease which might account for $50 \%$ of cases [15].

Due to its sub-acute course, the diagnosis of Pott's disease can be delayed or even go unrecognized for months. The late presentation was reported in both non-endemic areas where the incidence of the disease is low, and in the endemic area where the access to medical care could be limited [5] [8]. The delay in diagnosis and treatment is a major factor in determining the disease outcomes [16]. Thus, early diagnosis and treatment are essential keys to prevent devastating outcomes e.g. permanent neurological deficit or spinal deformity [1]. The newly developed laboratory and imaging modalities during these years and different treatment protocols mandate applying new guidelines to diagnose and treat this serious disease. New reviews are always required to assess modern diagnostic modalities and treatment options. 
The aim of this study is to review spinal Tuberculosis (TB) cases in our tertiary care center and evaluate it from different aspects, which may provide great support to the clinical decisions of this disease.

\section{Methodology}

The study is a retrospective charts review of all adult spinal tuberculosis patients (i.e. 14 years and older) who were evaluated at King Abdulaziz Medical City (KAMC) in Riyadh, Saudi Arabia, from January 2001 to March 2015.

The patient's charts were the main source of data and reviewed by using the ICD codes for Spinal Tuberculosis, Pott's disease, tuberculous spondylitis, and Vertebral TB through the medical records department.

Patients demography, presenting symptoms and signs, radiological and biological data for all the patients fulfilling a specific case definition were reviewed and analyzed.

Case definition: Any patient with symptoms or signs suggestive of spinal TB with supportive radiological evidence of infection and the diagnosis confirmed by either positive AFB tissue culture for mycobacterium tuberculosis or tissue biopsy showing pathological features of TB.

Inclusion criteria: All patients fulfilled the case definition and older than 14 years.

Exclusion criteria: Any patient diagnosed with spinal tuberculosis but not fulfill the case definition or younger than 14 years old.

The actual patient's identity was masked. The Ethical approval was obtained from King Abdullah Medical Research Center, Riyadh, Saudi Arabia.

\section{Results}

A total of 61 cases out of 120 screened charts were included according to the case definition. 36 (59\%) were female, and the mean age was 51.3 years with a range of 14 to 88 years old. Nine patients had a previous history of TB, 5 of them they had spinal TB, and the other 4 had pulmonary TB (Table 1 ).

The most presenting symptom is back pain in 52 cases $(85 \%)$, followed by lower limbs weakness in 32 cases (52\%), and lower limbs pain in 21 cases (34\%). Fever, night sweating and weight loss presented in 18 (30\%), 16 (26\%), and 13 $(21 \%)$ cases respectively (Figure 1$)$. The patients usually presented with chronic symptoms (mean 170 days) and the average duration of hospital stay for these patients was 41 days. On physical examination, 51\% had bilateral lower limbs weakness as in (Figure 2).

Acute phase reactants like CRP and ESR were high in most of the patients, $97 \%$ and $92 \%$ respectively, but leukocytosis was not common and seen only in $16 \%$ (Table 2).

Out of all the patients who had a biopsy done, 51 patients (86\%) out of 59 tested patients had positive AFB culture for mycobacterium tuberculosis. MTB PCR was done from spine biopsy in 16 patients, and $81 \%$ came positive as shown in (Table 3). Only one patient had multi-drug resistance TB with previous spinal 
TB infection.

In pathological description, 53 tissue biopsies done, 45\% showed necrotizing granuloma, $21 \%$ non-necrotizing granuloma, and $34 \%$ non-specific inflammation (Table 4).

Imaging (either CT scan or MRI) was performed on the patients at the time of presentation. As shown in (Table 5), the most common signs seen in both were vertebral bone destruction and perivertebral collections. The Thoracic/Lumbar vertebrae were the most common involved locations (Table 6). Thirty-one patients had both modalities done (CT and MRI), and spinal compression was seen on MRI in $68 \%$ of the cases compared to $36 \%$ on the CT scan with a P-value less than 0.05 (Table 5).

All patients received anti-tuberculous therapy (standard first line) with some individual adjustment according to the response and the complications, with an average of 15 months of treatment. 11 (18\%) patients developed side effects from the medications, 7 of them develop hepatitis, and two patients had vomiting, and one patient had vision loss, and one patient had increasing bilirubin. On the other hand, 24 (39\%) of the patients needed surgical intervention for spin TB complications and reached up to 27 spinal procedures (Table 7).



Figure 1. Presenting symptoms.

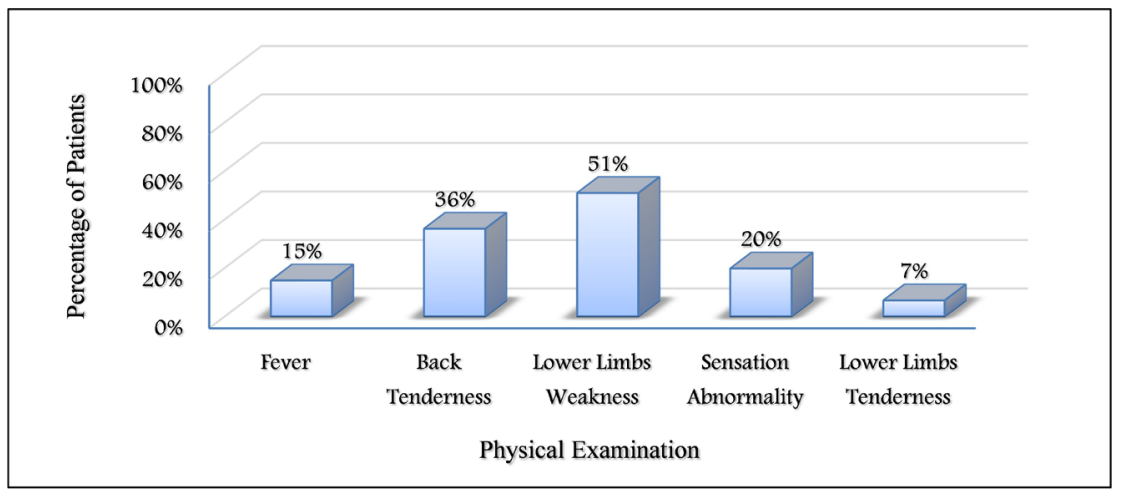

Figure 2. Physical examination. 
Table 1. Demographic data.

\begin{tabular}{|c|c|c|}
\hline \multicolumn{3}{|c|}{ Demographic Data } \\
\hline Factors & Number (\%) & \\
\hline Age, mean & 51.3 years & (From 14 to 88 years) \\
\hline \multicolumn{3}{|l|}{ Age distribution, years: } \\
\hline $14-30$ & $12(19.67 \%)$ & \\
\hline $31-60$ & $26(42.62 \%)$ & \\
\hline $61-88$ & $23(37.71 \%)$ & \\
\hline \multicolumn{3}{|l|}{ Gender } \\
\hline Male & $25(40.98 \%)$ & \\
\hline Female & $36(59.02 \%)$ & \\
\hline \multicolumn{3}{|l|}{ Nationality } \\
\hline Saudi & $59(96.72 \%)$ & \\
\hline Non-Saudi & $2(3.28 \%)$ & \\
\hline \multicolumn{3}{|l|}{ Medical Background } \\
\hline Diabetes mellitus & $15(24.59 \%)$ & \\
\hline Hypertension & $22(36.07 \%)$ & \\
\hline Chronic kidney disease & $5(8.20 \%)$ & \\
\hline Transplanted organ (Liver) & $1(1.64 \%)$ & \\
\hline Hepatitis $\mathrm{C}$ virus & $1(1.64 \%)$ & \\
\hline Hepatitis B virus & $1(1.64 \%)$ & \\
\hline Myelodysplastic syndrome & $1(1.64 \%)$ & \\
\hline \multicolumn{3}{|l|}{ History of previous TB } \\
\hline Pulmonary & $4(6.56 \%)$ & \\
\hline Spine & $5(8.20 \%)$ & \\
\hline Total & $9(14.75 \%)$ & \\
\hline Hospital Stay, mean & 41 days & (From 1 to 275 days) \\
\hline Duration of symptoms, mean & 170 days & (From 2 to 2190 days) \\
\hline
\end{tabular}

Table 2. Laboratory finding.

\begin{tabular}{ccc}
\hline Lab & Number $(\%)$ & Reference \\
\hline WBC & $10(16.39 \%)$ & WBC $>11.0 \times 10^{9} / \mathrm{L}$ \\
Leukocytosis & $3(4.92 \%)$ & WBC $<4.0 \times 10^{9} / \mathrm{L}$ \\
Leukopenia & & \\
Hemoglobin & $41(67.21 \%)$ & Hemoglobin $<135 \mathrm{gm} / \mathrm{L}$ \\
Anemia & & Normal ESR $0-20 \mathrm{~mm} / \mathrm{h}$ \\
Acute Phase Reactant & $50(92.59 \%)$ & Normal CRP $0-3.5 \mathrm{mg} / \mathrm{L}$ \\
High ESR n $=54$ & $35(97.22 \%)$ & Sodium $<136 \mathrm{mEq} / \mathrm{L}$ \\
High CRP n $=36$ & & \\
Sodium & $30(50 \%)$ & Platelets $>400 \times 10^{9} / \mathrm{L}$ \\
Hyponatremia $n=60$ & & Platelets $<150 \times 10^{9} / \mathrm{L}$ \\
Platelets & $2(3.28 \%)$ & \\
Thrombocytosis & & \\
Thrombocytopenia & & \\
\hline
\end{tabular}


Table 3. Microbiology.

\begin{tabular}{ccc}
\hline Test & Positive & Negative \\
\hline AFB Histopathology $\mathrm{n}=53$ & $2(3.77 \%)$ & $51(96.23 \%)$ \\
TB PCR $\mathrm{n}=16$ & $13(81.25 \%)$ & $3(18.75 \%)$ \\
TB Culture $\mathrm{n}=59$ & $51(86.44 \%)$ & $8(13.56 \%)$ \\
Culture susceptibility $\mathrm{n}=59$ & & \\
Pan Sensitive & $57(96.61 \%)$ & \\
MDR-MTB & $1(1.69 \%)$ & \\
\hline
\end{tabular}

Table 4. Tissue biopsies.

\begin{tabular}{cc}
\hline Tissue Biopsies $\boldsymbol{n}=\mathbf{5 3}$ & Number $(\%)$ \\
\hline Necrotizing granuloma (Caseating) & $24(45.28 \%)$ \\
Non-caseating granuloma & $11(20.75 \%)$ \\
Other (Inflammatory tissue, not specific) & $18(33.96 \%)$ \\
\hline
\end{tabular}

Table 5. Finding of the spinal images.

\begin{tabular}{|c|c|c|c|}
\hline Finding & \multicolumn{2}{|c|}{$C T n=40$, Number $(\%)$} & $M R I n=50$, Number $(\%)$ \\
\hline Spinal compression & \multicolumn{2}{|c|}{$13(32.50 \%)$} & $26(52 \%)$ \\
\hline $\begin{array}{c}\text { Collection } \\
\text { (Intraspinal/Intravertebral) }\end{array}$ & \multicolumn{2}{|c|}{$28(70 \%)$} & $42(84 \%)$ \\
\hline Paraspinal & \multicolumn{2}{|c|}{$9(32.14 \%)$} & $16(38.10 \%)$ \\
\hline Paravertebral & \multicolumn{2}{|c|}{$10(35.71 \%)$} & $23(54.76 \%)$ \\
\hline Epidural & \multicolumn{2}{|c|}{$5(17.86 \%)$} & $13(30.95 \%)$ \\
\hline Not specified & \multicolumn{2}{|c|}{$9(32.14 \%)$} & $2(4.76 \%)$ \\
\hline Vertebral destruction & \multicolumn{2}{|c|}{$38(95 \%)$} & $40(80 \%)$ \\
\hline Disc space & \multicolumn{2}{|c|}{$10(25 \%)$} & $19(38 \%)$ \\
\hline Other & \multicolumn{2}{|c|}{$11(27.50 \%)$} & $11(22 \%)$ \\
\hline Iliopsoas/Psoas collection & \multicolumn{2}{|c|}{$9(81.82 \%)$} & $3(27.27 \%)$ \\
\hline Lymphadenopathy & \multicolumn{2}{|c|}{$1(9.09 \%)$} & $1(9.09 \%)$ \\
\hline Pleural effusion & \multicolumn{2}{|c|}{$1(9.09 \%)$} & -- \\
\hline Gluteal abscess & \multicolumn{2}{|c|}{--} & $1(9.09 \%)$ \\
\hline Spinal mass/lesion & \multicolumn{2}{|c|}{--} & $3(27.27 \%)$ \\
\hline Bony deterioration & \multicolumn{2}{|c|}{--} & $1(9.09 \%)$ \\
\hline \multirow[t]{2}{*}{ Compression fracture } & \multicolumn{2}{|c|}{--} & $2(18.18 \%)$ \\
\hline & \multicolumn{2}{|c|}{$\begin{array}{c}C T \& M R I n=31 \\
\text { Number }(\%)\end{array}$} & \\
\hline Finding in both $C T$ and $M R I$ & $\underline{C T}$ & $\underline{M R I}$ & $\underline{P \text { Value }}$ \\
\hline Spinal compression & $11(35.58 \%)$ & $21(67.74 \%)$ & 0.0007 \\
\hline Collection & $21(67.74 \%)$ & $26(83.87 \%)$ & 0.0227 \\
\hline Vertebral destruction & $28(90.32 \%)$ & $27(87.10 \%)$ & 0.3253 \\
\hline Disc space & $8(25.81 \%)$ & $14(45.16 \%)$ & 0.0117 \\
\hline
\end{tabular}


Table 6. Site of spinal TB involvement.

\begin{tabular}{cc}
\hline Site of Involvement & Number $(\%)$ \\
\hline Cervical & $6(9.84 \%)$ \\
Thoracic & $18(29.51 \%)$ \\
Lumbar & $20(32.79 \%)$ \\
Sacral & $1(1.64 \%)$ \\
Cervical + Thoracic & $4(6.56 \%)$ \\
Cervical + Lumbar & $1(1.64 \%)$ \\
Thoracic + Lumbar & $10(16.67 \%)$ \\
Lumbar + Sacral & $1(1.64 \%)$ \\
\hline
\end{tabular}

Table 7. Management.

\begin{tabular}{cc}
\hline & Number (\%) \\
\hline Medication & $57(93.44 \%)$ \\
Isoniazid & $58(95.08 \%)$ \\
Pyrampicin & $60(98.36 \%)$ \\
Ethambutol & $60(98.36 \%)$ \\
Pyridoxine & $59(96.72 \%)$ \\
Moxifloxacin & $14(22.95 \%)$ \\
Ciprofloxacin & $5(8.20 \%)$ \\
Streptomycin & $1(1.64 \%)$ \\
Steroid & $14(22.95 \%)$ \\
Surgical Intervention, $\boldsymbol{n}=27$ Procedures & \\
Decompression (Laminectomy) & $24(88.87 \%)$ \\
Instrumental Stabilization & $15(55.56 \%)$ \\
Fusion (Bone grafting) & $3(11.11 \%)$ \\
\hline
\end{tabular}

\section{Discussion}

Spinal infection is a major dilemma that is difficult to confirm it and differentiate it clinically from other spinal diseases like tumors, degenerative changes, and non-inflammatory lesions despite improving different diagnostic methods [17].

Saudi Arabia considered as an endemic area of TB and spinal TB is quite common in our country as well as in developing countries [4] [18]. Alothman A et al. did a study in 69 patients from 1985 to 1998 at KAMC-Riyadh (which previously called King Fahad National Guard Hospital) and published in 2001 [4]. In their study, they found that the cases in the age group from 51 to 70 years was the highest group (52\%) which is almost like our finding (48\%). 
When we compare them to other different studies we found that the most age group are below 40 years as in Fareeha Rauf et al. with 90\% [1], Mehmet Turgut study with mean age of 32 years [18]. Also, an article review was done by E.M. Trecarichi et al., which includes 29 different observational studies showed a mean age from 30 to 40 years [19].

The most common presenting symptom in this study is back pain with $85 \%$ which is similar to the previous studies [19]. The second most common is lower limb weakness with $53 \%$. Fever and constitutional symptoms usually less than $40 \%$ and $30 \%$ respectively in most of the studies [19]. In a retrospective study done in China including 967 cases showed the Back pain was the most common symptom, followed by night sweats and low-grade fever [20].

Regarding physical examination, Neurological deficits usually caused by a spinal abscess or tissue compressing the spinal cord or cauda equina or spinal deformity like kyphosis [21]. As per the study by Colmenero et al. [19], 45\% of spinal TB cases had neurological deficits, and these patients were more likely to have epidural, paraspinal or psoas muscle abscesses. The most common neurological signs are lower limb weakness, numbness, and urinary disorders [22] [23] [24] [25]. No much difference in our results, 51\% had lower limbs weakness, and $20 \%$ had sensation abnormality. In contrast, Back tenderness is the most common physical finding in Alothman et al. study compared to our study which is the second, after the lower limb's weakness, with $45 \%$ and $36 \%$ respectively [ 4 ].

The duration of the complaint to the diagnosis may take a long time and this may reflect one of the difficulties that face the clinicians as can be ranged from 2 months to 4 years [19]. A case included here with six years of symptoms till the diagnosis. Also, four other cases complaint for about two years before the diagnosis done.

Although Alothman et al. study showed $73 \%$ of the cases with elevated ESR, $92 \%$ of our cases had elevated results. In another hand, leukocytosis was not common in both studies and showed only $16 \%$ in each study [4]. Interestingly, the C-reactive protein (CRP) has usually been found to be normal or slightly elevated in previous studies, but here it positive in $97 \%$ of the cases who investigated [19]. Chronic diseases can cause anemia, which was evident in $67 \%$ of the cases who were anemic.

24 patients underwent surgery, three of them underwent twice. The other patients (61\%), underwent for imaging-guided biopsies or aspirations which are not difficult and reasonable approach for the patient who doesn't need a surgery [26] [27]. TB cultures were positive in a good number of the patient (86\%) which is giving us an idea of its value. 53 tissue biopsies done, either during the surgery or by imaging-guided, showed granuloma in $66 \%$.

Typically, the site of TB spine involvement would be in the thoracic and lumbar regions most of the time with $89 \%$ of the cases, and it can affect the vertebrae, the intervertebral discs and the intravertebral space is by the extension of the abscess [4] [19] [28]. Here, the common involvement sites are reversed. So, 
the commonest site is lumber followed by thoracic then thoraco-lumber and finally cervical region with $33 \%, 30 \%, 17 \%$, and $10 \%$ respectively.

The most modality used for radiological investigation was spinal MRI followed by spinal CT scan with $82 \%$ and $66 \%$ respectively. The most common finding on imaging was the intravertebral collection and vertebral destruction. Regarding the cases who underwent for both modalities, spinal compression, intravertebral collection, and disc space involvement are better recognized by MRI than CT scan with significant $P$ values.

There is no statistically significant difference regarding the vertebral destruction. So, as also concluded in other previous studies [1] [4] [19] [28], MRI is a better imaging modality to provide a picture of spinal TB.

Finally, all the patients received the first line of anti-TB (isoniazid, rifampicin, ethambutol, and pyrazinamide) with a mean duration of 15 months. According to the Infectious Disease Society Guidelines for the treatment of drug-susceptible $\mathrm{TB}$, the duration of therapy of spinal $\mathrm{TB}$ is six to nine months with regimens including rifampicin which is equal to 18 months' treatment with regimens not including it. Also, uncomplicated spinal TB can be treated medically without surgery unless the patient has recurrent or persistence neurological deficit secondary to cord compression, his condition not improving with anti-TB or instability of the spine [28].

Future research questions need to emphasizeon early identification of Tuberculous Spondylitis and differentiating it from other inflammatory and degenerative spinal diseases [29]. Access of ambulatory patients with suspected spinal pain to MRI or CT scan can be delayed for months. Developing a scoring system that combines clinical and basic laboratory tests may help to prioritize early access to further sensitive diagnostics. Identification of early immunological markers of active TB infection such as IGRA or T SPOT and differentiation it from to latent TB infections was difficulty to be established. However qualitative immunological markers may provide support to early diagnostic even at the stage of osteitis prior to the development of paraspinal abscess and complicated diseased [30].

The limitations of this study are being retrospective analysis and the charts, notdigitals. So, some clinical symptoms or signs may be missed. In addition, the sample size is quietly low as the study was done in a single tertiary center.

\section{Conclusion}

Spinal Tuberculosis is a chronic presentation illness with a non-specific clinical picture. Interventional diagnostics is the best current modality to reach a confirmatory diagnosis. The gold standard for confirming TB diagnosis probably will remain cultures in addition to early utilization of molecular TB diagnostics. Time for diagnosis of Spinal Tuberculosis is delayed despite improvement in diagnostics tests. Medical treatment is the major solution of uncomplicated spinal TB. 


\section{Conflicts of Interest}

The authors declare no conflicts of interest regarding the publication of this paper.

\section{References}

[1] Rauf, F., Chaudhry, U.R., Atif, M. and ur Rahaman, M. (2015) Spinal Tuberculosis: Our Experience and a Review of Imaging Methods. The Neuroradiology Journal, 28, 498-503. https://doi.org/10.1177/1971400915609874

[2] MOH Statistical Year Book-1437. Saudi Ministry of Health, Saudi Arabia, 2016. https://www.moh.gov.sa/en/Ministry/Statistics/book/Documents/Statistical-Yearbo ok-1437H.pdf

[3] World Health Organization (2017) Global Tuberculosis Report 2017. World Health Organization, Geneva. http://www.who.int/tb/publications/global_report/en/

[4] Alothman, A., Memish, Z.A., Awada, A., et al. (2001) Tuberculous Spondylitis: Analysis of 69 Cases from Saudi Arabia. Spine, 26, E565-E570. https://doi.org/10.1097/00007632-200112150-00020

[5] Fuentes Ferrer, M., Gutiérrez Torres, L., Ayala Ramírez, O., Rumayor Zarzuelo, M. and del Prado González, N. (2012) Tuberculosis of the Spine. A Systematic Review of Case Series. International Orthopaedics, 36, 221-231. https://doi.org/10.1007/s00264-011-1414-4

[6] Hodgson, S.P. and Ormerod, L.P. (1990) Ten-Year Experience of Bone and Joint Tuberculosis in Blackburn 1978-1987. Journal of the Royal College of Surgeons of Edinburgh, 35, 259-262.

[7] Hopewell, P.C. (1994) Overview of Clinical Tuberculosis. Tuberculosis, January, 25-46.

[8] Nussbaum, E.S., Rockswold, G.L., Bergman, T.A., Erickson, D.L. and Seljeskog, E.L. (1995) Spinal Tuberculosis: A Diagnostic and Management Challenge. Journal of Neurosurgery, 83, 243-247. https://doi.org/10.3171/jns.1995.83.2.0243

[9] Pigrau-Serrallach, C. and Rodríguez-Pardo, D. (2013) Bone and Joint Tuberculosis. European Spine Journal, 22, 556-566. https://doi.org/10.1007/s00586-012-2331-y

[10] Fanning, A. (1999) Tuberculosis: 6. Extrapulmonary Disease. Journal of the Oklahoma State Medical Association, 160, 1597-1603.

[11] Peto, H.M., Pratt, R.H., Harrington, T.A., LoBue, P.A. and Armstrong, L.R. (2009) Epidemiology of Extrapulmonary Tuberculosis in the United States, 1993-2006. Clinical Infectious Diseases, 49, 1350-1357. https://doi.org/10.1086/605559

[12] Sharma, S.K. and Mohan, A. (2004) Extrapulmonary Tuberculosis. The Indian Journal of Medical Research, 120, 316-353.

[13] Teo, H.E.L. and Peh, W.C.G. (2004) Skeletal Tuberculosis in Children. Pediatric Radiology, 34, 853-860. https://doi.org/10.1007/s00247-004-1223-7

[14] Watts, H.G. and Lifeso, R.M. (1996) Tuberculosis of Bones and Joints. The Journal of Bone and Joint Surgery-American Volume, 78, 288-299. https://doi.org/10.2106/00004623-199602000-00019

[15] Vohra, R., Kang, H.S., Dogra, S., Saggar, R.R. and Sharma, R. (1997) Tuberculous Osteomyelitis. The Journal of Bone and Joint Surgery-British Volume, 79, 562-566. https://doi.org/10.1302/0301-620X.79B4.7618

[16] Kamara, E., Mehta, S., Brust, J.C.M. and Jain, A.K. (2012) Effect of Delayed Diagnosis on Severity of Pott's Disease. International Orthopaedics, 36, 245-254. 
https://doi.org/10.1007/s00264-011-1432-2

[17] Sharif, H.S. (1992) Role of MR Imaging in the Management of Spinal Infections. American Journal of Roentgenology, 158, 1333-1345.

https://doi.org/10.2214/ajr.158.6.1590137

[18] Turgut, M. (2001) Spinal Tuberculosis (Pott's Disease): Its Clinical Presentation, Surgical Management, and Outcome. A Survey Study on 694 Patients. Neurosurgical Review, 24, 8-13. https://doi.org/10.1007/PL00011973

[19] Trecarichi, E.M., Meco, E.D., Mazzotta, V. and Fantoni, M. (2012) Tuberculous Spondylodiscitis: Epidemiology, Clinical Features, Treatment, and Outcome. European Review for Medical and Pharmacological Sciences, 2, 58-72.

[20] Shi, T., Zhang, Z.H., Dai, F., et al. (2016) Retrospective Study of 967 Patients with Spinal Tuberculosis. Orthopedics, 39, E838-E843. https://doi.org/10.3928/01477447-20160509-03

[21] Ailinger, R.L., Martyn, D., Lasus, H. and Lima Garcia, N. (2010) The Effect of a Cultural Intervention on Adherence to Latent Tuberculosis Infection Therapy in Latino Immigrants. Public Health Nursing, 27, 115-120. https://doi.org/10.1111/j.1525-1446.2010.00834.x

[22] Hadadi, A., Rasoulinejad, M., Khashayar, P., Mosavi, M. and Maghighi Morad, M. (2010) Osteoarticular Tuberculosis in Tehran, Iran: A 2-Year Study. Clinical Microbiology and Infection, 16, 1270-1273. https://doi.org/10.1111/j.1469-0691.2009.03082.x

[23] Mwachaka, P.M., Ranketi, S.S., Nchafatso, O.G., Kasyoka, B.M. and Kiboi, J.G. (2011) Spinal Tuberculosis among Human Immunodeficiency Virus-Negative Patients in a Kenyan Tertiary Hospital: A 5-Year Synopsis. The Spine Journal, 11, 265-269. https://doi.org/10.1016/j.spinee.2011.01.033

[24] Rezai, A.R., Lee, M., Cooper, P.R., Errico, T.J. and Koslow, M. (1995) Modern Management of Spinal Tuberculosis. Neurosurgery, 36, 87-98; Discussion 97-98. https://doi.org/10.1227/00006123-199501000-00011

[25] Solagberu, B.A. and Ayorinde, R.O. (2001) Tuberculosis of the Spine in Ilorin, Nigeria. East African Medical Journal, 78, 197-199. https://doi.org/10.4314/eamj.v78i4.9062

[26] Francis, I.M., Das, D.K., Luthra, U.K., Sheikh, Z., Sheikh, M. and Bashir, M. (1999) Value of Radiologically Guided Fine Needle Aspiration Cytology (FNAC) in the Diagnosis of spinal Tuberculosis: A Study of 29 Cases. Cytopathology, 10, 390-401. https://doi.org/10.1046/j.1365-2303.1999.00206.x

[27] Mondal, A. (1994) Cytological Diagnosis of Vertebral Tuberculosis with Fine-Needle Aspiration Biopsy. The Journal of Bone and Joint Surgery-American Volume, 76, 181-184. https://doi.org/10.2106/00004623-199402000-00003

[28] Nahid, P., Dorman, S.E., Alipanah, N., et al. (2016) Official American Thoracic Society/Centers for Disease Control and Prevention/Infectious Diseases Society of America Clinical Practice Guidelines: Treatment of Drug-Susceptible Tuberculosis. Clinical Infectious Diseases, 63, E147-E195. https://doi.org/10.1093/cid/ciw376

[29] Chen, C.-H., Chen, Y.-M., Lee, C.-W., Chang, Y.-J., Cheng, C.-Y. and Hung, J.-K. (2016) Early Diagnosis of Spinal Tuberculosis. Journal of the Formosan Medical Association, 115, 825-836. https://doi.org/10.1016/j.jfma.2016.07.001

[30] Whitworth, H.S., Scott, M., Connell, D.W., Dongés, B. and Lalvani, A. (2013) IGRAs-The Gateway to T Cell Based TB Diagnosis. Methods, 61, 52-62. https://doi.org/10.1016/j.ymeth.2012.12.012 


\section{List of Abbreviations}

Tuberculosis (TB): Mycobacterium tuberculosis (MTB)

White blood cells (WBC): Acid-fast bacilli (AFB)

Polymerase chain reaction (PCR): International Classification of Diseases (ICD)

Computed tomography (CT): Magnetic resonance imaging (MRI)

Interferon gamma release assay (IGRA): C-reactive protein (CRP)

Erythrocyte sedimentation rate (ESR) 